\title{
Über Sturzmelder und andere Pflegeroboter
}

\author{
Wolff, Eberhard
}

\begin{abstract}
Falls Sie nicht wissen, was ein «Hubot» ist, empfiehlt sich die Lektüre dieses Beitrags ganz besonderes. Denn er kann unheimlich hilfreich sein: für Opi Tee und Spaghetti kochen oder ihn bei Bedarf reanimieren. Äusserst vielseitig also. Wie Mensch und Technik interagieren, untersuchte ein Tagung an der Universität Fribourg.
\end{abstract}

Posted at the Zurich Open Repository and Archive, University of Zurich ZORA URL: https://doi.org/10.5167/uzh-92261

Journal Article

Published Version

Originally published at:

Wolff, Eberhard (2013). Über Sturzmelder und andere Pflegeroboter. Schweizerische Ärztezeitung (SÄZ), 94(49):1884. 


\section{Über Sturzmelder und andere Pflegeroboter}

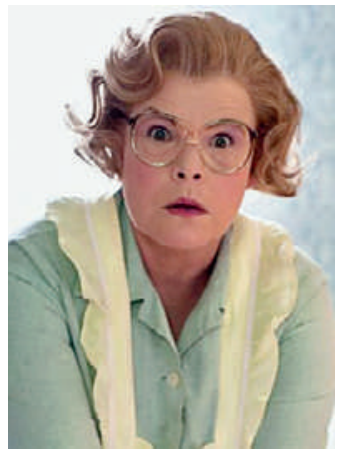

Vera, der Pflege-«Hubot». Foto: SRF
* Die Referenzen finden sich im Internet unter www.saez.ch $\rightarrow$ Aktuelle Ausgabe oder $\rightarrow$ Archiv $\rightarrow 2013 \rightarrow 49$.

** PD Dr. rer. soc. Eberhard Wolff ist Kulturwissenschaftler, Medizinhistoriker und Mitglied der Redaktion Medizingeschichte der Schweizerischen Ärztezeitung.
Ich weiss, ich weiss: Ein Sturzmelder ist eigentlich kein Pflegeroboter, eher ein technisches Hilfsmittel für allein lebende ältere und gebrechliche Menschen, für die ein nicht entdeckter Sturz tödliche Folgen haben kann. In der Schweiz trifft dies jedes Jahr etwa 1000 Personen. Das immer unauffälligere Gerät am Körper erkennt den Bewegungsablauf eines Sturzes oder Zusammensinkens von alleine und gibt Alarm, zum Beispiel über das Hausnotrufgerät. Ein Roboter dagegen ist komplex, agiert autonom und interagiert mit dem Menschen. Aber ein Stück weit passt das auch auf den Sturzmelder. Die Grenzen zerfliessen eben zunehmend.

Das Schweizer Fernsehen hat kürzlich die schwedische Serie «Real Humans» ausgestrahlt, die in einer Zeit spielt, in der humanoide Roboter («Hubots») im Alltag helfen. Der allein lebende Opa Lennart hat zum Beispiel Odi, seinen Hubot-Kumpel, der mit ihm spielt, für ihn Auto fährt und ihm Lasagne kocht. Als Odi kaputtgeht, wird er von Lennarts Kindern durch die klischeehaft strenge Hubot-Pflegerin Vera ersetzt (siehe Photo), die ihm Tee aufzwingt und den Alkohol verbietet, ihn beim Infarkt aber auch reanimiert. Das hätte selbst der intelligenteste heutige Sturzmelder nicht alleine zuwege gebracht.

In der Serie glauben die Menschen, die Robotertechnik einfach nur für ein angenehmeres Leben einsetzen zu können. Doch plötzlich verändern die «Hubots» auch die Menschen, nicht nur Lennarts Lasagne- und Weinumsatz. Mit einem Hubot neben sich beginnen die Menschen anders zu denken und anders zu handeln [1].*

Radikaler Szenenwechsel: Im tristen Beton der Universität Fribourg trafen sich diesen Sommer auf Einladung der SAGW Gesundheitsforscher (nur wirklich echte Menschen), die mögliche Beiträge der Geisteswissenschaften zur Lösung anstehender Herausforderungen in der Medizin ausloten wollten [2]. Ein Workshop griff das zurzeit heisse Thema [3] unter dem kühlen Titel «Technologie und selbständige Lebensführung» auf. In den Vorträgen der beiden Referierenden kamen sich Pflegeroboter und Sturzmelder dann bedrohlich nahe.

Dr. Luigi Corrado leitet die ARBT, einen Zusammenschluss von Institutionen der Hausbetreuung, die in der Romandie gerontotechnische Services wie Sturzmelder, Notruftelefone oder Ortungsdienste anbieten [4]. Das läuft dort auch unter dem Begriff der «biotélévigilance», den man besser gar nicht erst zu übersetzen versucht. Die andere Referentin, Prof. Dr. Heidrun Becker von der ZHAW, ist Mitautorin einer Studie von TA-Swiss über Robotik in der Gesund- heitsversorgung, die den kommenden Handlungsbedarf abschätzen sollte [5].

Ein Aspekt der Vorträge: Pflegeroboter oder Sturzmelder sollen nicht nur Geld und Arbeitskraft in der Pflege sparen, sie sollen auch antreten, die Autonomie und Mobilität älterer Menschen zu erhalten: ein Plus an Lebensqualität. Pflegetechnik kann aber auch das Gegenteil bewirken. Befragte äussern die Befürchtung, dass die Pflegerobotik zwischenmenschliche Kontakte reduziert und ein Gefühl des Überwachtseins vermittelt: ein Verlust an Autonomie, Würde, Fürsorge, und damit ein Minus an Lebensqualität.

Geisteswissenschaftler können von aussen manchmal mehr sehen als andere, auch Unangenehmes. Deshalb stehen sie im schlechten Ruf, bei neuer Technik vor allem «Bedenkenträger» zu sein. Manche Geisteswissenschaftler arbeiten diesem Image aber entgegen. Bevor sie wertend nur Chancen und Risiken bestimmen, wollen sie erst einmal verstehen, was im Umgang mit neuer Technik abläuft. Und das hat auch viel mit den Hubots zu tun.

Mit einem Sturzmelder ändern sich die Menschen. Wer ihn trägt, kommt sich vielleicht plötzlich älter vor, bewegt sich aber vielleicht auch selbstsicherer. Findige setzen ihn womöglich subversiv ein, damit mal jemand vorbeikommt. Für Angehörige kann er eine grosse Entlastung sein, einerseits ein Grund, seltener zu Besuch zu kommen, andererseits aber auch die Chance, freiwilliger, spontaner mal vorbeizuschauen [6].

Geräte haben immanente Werte, meinte in seiner Einführung der Workshopleiter Thomas Hengartner, Professor für Populäre Kulturen an der Universität Zürich und Spezialist in Technik-Kulturen. Geräte «schreiben» sich in die Menschen ein. Mensch und Technik stehen sich nicht gegenüber, sondern interagieren, vermischen sich. Dies mit fundamentalen Auswirkungen. Soziale und kulturelle Ordnungen werden von der Technik durchwirkt, auch von einem Sturzmelder oder bald mal einem Roboter. Wir tragen eine Technik wie den Sturzmelder nicht nur auf dem Körper, sondern auch «im Kopf». Und mal ehrlich: Denken Sie Ihre Vorträge nicht auch ein wenig powerpointisch, seit Sie mit dem Programm arbeiten? Diese Gedanken sind, vereinfacht gesagt, die Grundlage einer derzeit überaus attraktiven Theorie des französischen Soziologen Bruno Latour [7].

Und in dieser Hinsicht hat unsere fantastische Roboter-Pflegerin Vera mit ihrem eindringlichen Blick doch mehr mit einem Sturzmelder gemein, als man zunächst denkt.

Eberhard Wolff** 


\section{Referenzen}

1 Der gleiche Gedanke liegt im Übrigen auch dem neuen Kinofilm «Robot \& Frank» (mit Susan Sarandon) zugrunde.

2 www.sagw.ch/sagw/laufende-projekte/gesundheit/ tagung

3 Siehe eine einschlägige Klausurwoche kürzlich an der Universität Ulm: www.uni-ulm.de/med/med-medgeschichte/oeffentliche-veranstaltungen/tagungenworkshops/bmbf-klausurwoche-technisierung-des-alters.html
4 www.arbt.ch

5 www.ta-swiss.ch/robotik/

6 Plastisch illustriert in dem Produktwerbefilm für den «Butler» auf www.rettung-auf-knopfdruck.de. Andere Sturzmelder-Anbieter sind z.B. www.vigilio.eu oder www.tunstall.ch

7 Die sogenannte «Akteur-Netzwerk-Theorie» (ANT). 